\title{
ECONOMIC EFFICIENCY ON OVERSEEDING GRASSLANDS FROM PREAJBA - GORJ COUNTY IN 2014
}

\author{
DRAGOS MIHAI Medelete \\ assistant professor / Ph.D., Faculty of Agriculture AND Horticulture/Department " Land measurements \\ management, mechanization." University of Craiova, Craiova, Romania, medelete@yahoo.com \\ RADU LUCIAN Pânzaru \\ associate professor / Ph.D., Faculty of Agriculture AND Horticulture/Department " Land measurements \\ management, mechanization." University of Craiova, Craiova, Romania, rlp1967craiova@yahoo.com
}

\begin{abstract}
This study was achieved using the support of strategic project "Support Scholarships University in Romania by the European Doctoral and Post-doctoral (SCHOLARSHIPS DOC-POSTDOC)", ID 133255. The paper emphasizes the importance of meadows and hayfields production, considering that their value can be increased using over-seeding and organic-mineral fertilizers. Experience is located in the Experimental Center for Meadows Culture -Preajba, Gorj County, on natural meadow of Agrostis capillaris, over-seeded with Red clover. The production have increase with fertilizer dose, but maximum economic efficiency was found on variant that use only organically fertilizer. It should be noted that organic fertilizers are used in the first year only partially by plants.

The indicators of economic efficiency used are: raw product, variable costs, fixed costs, production costs, and the indices: total expenditure rate of profit, income taxes, net profit and net profit rate.
\end{abstract}

Key words: pastures, manure, fertilizers, profit, gross profit margin

\section{Introduction}

Permanent grasslands of Agrostis capillaris can be may be used as grazing and hay fields, hay obtained is of average quality. By over-seeding with Red clover and utilization of manure, pasture quality increase and other valuable leguminous reappear [1].

In developing culture technologies is follow the maximizing yields per hectare, avoiding depreciation of feed quality, increased mechanization of work, labor productivity growth, reducing the unitary cost [4].

Farmers can use better the manure obtained in their household and must bear in mind that this fertilizer is green and a good plant enhancer [2].

Natural pastures and hayfields have shares of $22 \%$ and $10 \%$ of agricultural land, and taking into account the distribution of land suitability classes, that only $17.8 \%$ of natural grasslands belong to Classes I - III, which suggests measures to improve the land occupied by these uses [3].

The goal of this study is to determine which of the variants of fertilization is better in terms of economic, using a set of indicators (raw product, variable costs, fixed costs, production costs, and the indices: total expenditure rate of profit, income taxes, net profit and net profit rate) .

\section{Economic efficiency}

For determining the efficiency a number of 8 variants are used, arranged in 3 repetitions:

Var. 1 = unfertilized;

Var. $2=5 \mathrm{t} /$ ha manure;

Var. $3=10$ t/ha manure;

Var. $4=15 \mathrm{t} / \mathrm{ha}$ manure;

Var. $5=20$ t/ha manure;

Var. $6=10 \mathrm{t} / \mathrm{ha} \mathrm{manure}+50 \mathrm{~kg} / \mathrm{ha} \mathrm{N}+50 \mathrm{~kg} / \mathrm{ha} \mathrm{P}_{2} \mathrm{O}_{5}+50 \mathrm{~kg} / \mathrm{ha} \mathrm{K}_{2} \mathrm{O}$; 
Var. $7=15 \mathrm{t} / \mathrm{ha}$ manure $+50 \mathrm{~kg} / \mathrm{ha} \mathrm{N}+50 \mathrm{~kg} / \mathrm{ha} \mathrm{P}_{2} \mathrm{O}_{5}+50 \mathrm{~kg} / \mathrm{ha} \mathrm{K}_{2} \mathrm{O}$;

Var. $8=20$ t/ha manure $+50 \mathrm{~kg} / \mathrm{ha} \mathrm{N}+50 \mathrm{~kg} / \mathrm{ha} \mathrm{P}_{2} \mathrm{O}_{5}+50 \mathrm{~kg} / \mathrm{ha} \mathrm{K}_{2} \mathrm{O}$.

The crude product was made by adding of grants (amounting to 156.89 euro / ha) to the main production,

Table 1 shows the economic indicators value on each variant used, main production being maximum (3556 lei) for variant 7, and minimum for variant 1 (1572 lei). In this condition the total production expenses growth from 877, 28 lei (variant 1) to 1449, 63 lei (variant 8).

Table 1: Economic efficiency of natural grassland of Agrostis capillaris, over-seeded with Red clover in 2014

\begin{tabular}{|c|c|c|c|c|c|}
\hline No. & Specification & Variant & $\begin{array}{l}\text { Quantity } \\
\text { tones, } \mathrm{kg} \text {, } \\
\text { liters }\end{array}$ & $\begin{array}{l}\text { Unitary } \\
\text { price } \\
\text { Lei }\end{array}$ & $\begin{array}{l}\text { Value } \\
\text { Lei }\end{array}$ \\
\hline 1. & $\begin{array}{c}\text { CRUDE } \\
\text { PRODUCT }\end{array}$ & - & - & - & \\
\hline \multirow{8}{*}{1.1.} & \multirow{8}{*}{$\begin{array}{c}\text { Main } \\
\text { production } \\
\text { (average yield - } \\
\text { kg/ha) }\end{array}$} & unfertilized & 3,93 & 400 & 1572 \\
\hline & & $5 \mathrm{t} / \mathrm{ha}$ manure & 4,75 & 400 & 1896 \\
\hline & & 10 t/ha manure & 5,56 & 400 & 2224 \\
\hline & & 15 t/ha manure & 5,97 & 400 & 2388 \\
\hline & & $20 \mathrm{t} / \mathrm{ha}$ manure & 7,51 & 400 & 3004 \\
\hline & & $10 \mathrm{t} /$ ha manure $+50 \mathrm{~N} 50 \mathrm{P}_{2} \mathrm{O}_{5} 50 \mathrm{~K}_{2} \mathrm{O}$ & 8,31 & 400 & 3324 \\
\hline & & $15 \mathrm{t} /$ ha manure $+50 \mathrm{~N} 50 \mathrm{P}_{2} \mathrm{O}_{5} 50 \mathrm{~K}_{2} \mathrm{O}$ & 8,89 & 400 & 3556 \\
\hline & & $20 \mathrm{t} /$ ha manure $+50 \mathrm{~N} 50 \mathrm{P}_{2} \mathrm{O}_{5} 50 \mathrm{~K}_{2} \mathrm{O}$ & 8,22 & 400 & 3288 \\
\hline 1.2 . & $\begin{array}{l}\text { Secondary } \\
\text { production - } \\
\text { kg/ha }\end{array}$ & - & - & - & \\
\hline \multirow{8}{*}{1.3.} & \multirow{8}{*}{$\begin{array}{l}\text { Gross product } \\
\text { from the } \\
\text { marketable } \\
\text { production }\end{array}$} & unfertilized & & & 1572 \\
\hline & & $5 \mathrm{t} / \mathrm{ha}$ manure & & & 1896 \\
\hline & & 10 t/ha manure & & & 2224 \\
\hline & & $15 \mathrm{t} / \mathrm{ha}$ manure & & & 2388 \\
\hline & & $20 \mathrm{t} / \mathrm{ha}$ manure & & & 3004 \\
\hline & & $10 \mathrm{t} /$ ha manure $+50 \mathrm{~N} 50 \mathrm{P}_{2} \mathrm{O}_{5} 50 \mathrm{~K}_{2} \mathrm{O}$ & & & 3324 \\
\hline & & $15 \mathrm{t} /$ ha manure $+50 \mathrm{~N} 50 \mathrm{P}_{2} \mathrm{O}_{5} 50 \mathrm{~K}_{2} \mathrm{O}$ & & & 3556 \\
\hline & & $20 \mathrm{t} /$ ha manure $+50 \mathrm{~N} 50 \mathrm{P}_{2} \mathrm{O}_{5} 50 \mathrm{~K}_{2} \mathrm{O}$ & & & 3288 \\
\hline 1.4. & Subsidies & $156,89 € /$ ha, $1 €=4,44$ lei & - & & 696,59 \\
\hline \multirow{8}{*}{1.5 . } & \multirow{8}{*}{$\begin{array}{c}\text { Total gross } \\
\text { product, including } \\
\text { subsidies }\end{array}$} & unfertilized & & & 2268,59 \\
\hline & & $5 \mathrm{t} / \mathrm{ha}$ manure & & & 2592,59 \\
\hline & & 10 t/ha manure & & & 2920,59 \\
\hline & & $15 \mathrm{t} / \mathrm{ha}$ manure & & & 3084,59 \\
\hline & & 20 t/ha manure & & & 3700,59 \\
\hline & & $10 \mathrm{t} /$ ha manure $+50 \mathrm{~N} 50 \mathrm{P}_{2} \mathrm{O}_{5} 50 \mathrm{~K}_{2} \mathrm{O}$ & & & 4020,59 \\
\hline & & $15 \mathrm{t} /$ ha manure $+50 \mathrm{~N} 50 \mathrm{P}_{2} \mathrm{O}_{5} 50 \mathrm{~K}_{2} \mathrm{O}$ & & & 4252,59 \\
\hline & & $20 \mathrm{t} / \mathrm{ha}$ manure $+50 \mathrm{~N} 50 \mathrm{P}_{2} \mathrm{O}_{5} 50 \mathrm{~K}_{2} \mathrm{O}$ & & & 3984,59 \\
\hline 2. & $\begin{array}{l}\text { VARIABLE } \\
\text { EXPENSES }\end{array}$ & & & & - \\
\hline 2.1 . & $\begin{array}{c}\text { Seeds } \\
\text { Dose - kg/ha }\end{array}$ & & 15 & 20 & 300 \\
\hline \multirow{6}{*}{2.2} & Fertilizers & unfertilized & - & - & - \\
\hline & $\begin{array}{c}\text { Fertilizers, } \\
\text { manure }\end{array}$ & 5 t/ha manure & 5 & 10 & 50 \\
\hline & $\begin{array}{c}\text { Fertilizers, } \\
\text { manure }\end{array}$ & $10 \mathrm{t} / \mathrm{ha}$ manure & 10 & 10 & 100 \\
\hline & $\begin{array}{c}\text { Fertilizers, } \\
\text { manure }\end{array}$ & 15 t/ha manure & 15 & 10 & 150 \\
\hline & $\begin{array}{c}\text { Fertilizers, } \\
\text { manure }\end{array}$ & 20 t/ha manure & 20 & 10 & 200 \\
\hline & $\begin{array}{c}\text { Fertilizers } \\
\text { NPK (15:15:15) } \\
\text { manure }\end{array}$ & $10 \mathrm{t} /$ ha manure $+50 \mathrm{~N} 50 \mathrm{P}_{2} \mathrm{O}_{5} 50 \mathrm{~K}_{2} \mathrm{O}$ & $\begin{array}{l}150 \mathrm{~kg} \\
10 \text { tone }\end{array}$ & $\begin{array}{c}2 \\
10\end{array}$ & 400 \\
\hline
\end{tabular}




\begin{tabular}{|c|c|c|c|c|c|}
\hline & $\begin{array}{c}\text { Fertilizers } \\
\text { NPK (15:15:15) } \\
\text { manure }\end{array}$ & $15 \mathrm{t}$ /ha manure $+50 \mathrm{~N} 50 \mathrm{P}_{2} \mathrm{O}_{5} 50 \mathrm{~K}_{2} \mathrm{O}$ & $\begin{array}{l}150 \mathrm{~kg} \\
15 \text { tons }\end{array}$ & $\begin{array}{c}2 \\
10\end{array}$ & 450 \\
\hline & $\begin{array}{c}\text { Fertilizers } \\
\text { NPK (15:15:15) } \\
\text { manure }\end{array}$ & $20 \mathrm{t} /$ ha manure $+50 \mathrm{~N} 50 \mathrm{P}_{2} \mathrm{O}_{5} 50 \mathrm{~K}_{2} \mathrm{O}$ & $\begin{array}{l}150 \mathrm{~kg} \\
20 \text { tons }\end{array}$ & $\begin{array}{c}2 \\
10\end{array}$ & 500 \\
\hline \multirow{8}{*}{2.3 . } & \multirow{8}{*}{$\begin{array}{c}\text { Mechanical works } \\
\text { with own means } \\
\text { (diesel / } \\
\text { lubricants, spare } \\
\text { parts, repair) }\end{array}$} & unfertilized & 36 liters & $\begin{array}{c}6 \\
15\end{array}$ & 231 \\
\hline & & 5 t/ha manure & 37 liters & $\begin{array}{c}6 \\
15\end{array}$ & 237 \\
\hline & & 10 t/ha manure & 38 liters & $\begin{array}{c}6 \\
15 \\
\end{array}$ & 243 \\
\hline & & $15 \mathrm{t} /$ ha manure & 38 liters & $\begin{array}{c}6 \\
15\end{array}$ & 243 \\
\hline & & 20 t/ha manure & 39 liters & $\begin{array}{c}6 \\
15 \\
\end{array}$ & 249 \\
\hline & & $10 \mathrm{t} /$ ha manure $+50 \mathrm{~N} 50 \mathrm{P}_{2} \mathrm{O}_{5} 50 \mathrm{~K}_{2} \mathrm{O}$ & 40 liters & $\begin{array}{c}6 \\
15\end{array}$ & 255 \\
\hline & & $15 \mathrm{t} / \mathrm{ha}$ manure $+50 \mathrm{~N} 50 \mathrm{P}_{2} \mathrm{O}_{5} \quad 50 \mathrm{~K}_{2} \mathrm{O}$ & 40 liters & $\begin{array}{c}6 \\
15 \\
\end{array}$ & 255 \\
\hline & & $20 \mathrm{t} / \mathrm{ha}$ manure $+50 \mathrm{~N} 50 \mathrm{P}_{2} \mathrm{O}_{5} 50 \mathrm{~K}_{2} \mathrm{O}$ & 40 liters & $\begin{array}{c}6 \\
15 \\
\end{array}$ & 255 \\
\hline 2.4 . & Crop insurance & - & - & & 40 \\
\hline 2.5 . & $\begin{array}{c}\text { Seasonal labor } \\
\text { expenses }\end{array}$ & - & 3 Z.O. & 60 & 180 \\
\hline \multirow{8}{*}{2.6} & \multirow{8}{*}{$\begin{array}{c}\text { TOTAL } \\
\text { VARIABLE } \\
\text { EXPENSES }\end{array}$} & unfertilized & & & 751 \\
\hline & & 5 t/ha manure & & & 807 \\
\hline & & $10 \mathrm{t} / \mathrm{ha}$ manure & & & 963 \\
\hline & & $15 \mathrm{t} / \mathrm{ha}$ manure & & & 919 \\
\hline & & $20 \mathrm{t} / \mathrm{ha}$ manure & & & 969 \\
\hline & & $10 \mathrm{t} /$ ha manure $+50 \mathrm{~N} 50 \mathrm{P}_{2} \mathrm{O}_{5} 50 \mathrm{~K}_{2} \mathrm{O}$ & & & 1175 \\
\hline & & $15 \mathrm{t} /$ ha manure $+50 \mathrm{~N} 50 \mathrm{P}_{2} \mathrm{O}_{5} 50 \mathrm{~K}_{2} \mathrm{O}$ & & & 1225 \\
\hline & & $20 \mathrm{t} /$ ha manure $+50 \mathrm{~N} 50 \mathrm{P}_{2} \mathrm{O}_{5} 50 \mathrm{~K}_{2} \mathrm{O}$ & & & 1275 \\
\hline 3. & $\begin{array}{c}\text { FIXED } \\
\text { EXPENSES }\end{array}$ & & & & - \\
\hline \multirow{8}{*}{ 3.1. } & \multirow{8}{*}{ 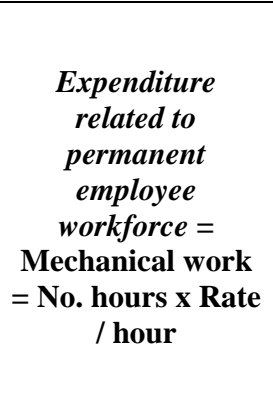 } & unfertilized & 10 & 10 & 100 \\
\hline & & 5 t/ha manure & 11 & 10 & 110 \\
\hline & & 10 t/ha manure & 11 & 10 & 110 \\
\hline & & $15 \mathrm{t} / \mathrm{ha}$ manure & 11 & 10 & 110 \\
\hline & & 20 t/ha manure & 12 & 10 & 120 \\
\hline & & $10 \mathrm{t} /$ ha manure $+50 \mathrm{~N} 50 \mathrm{P}_{2} \mathrm{O}_{5} 50 \mathrm{~K}_{2} \mathrm{O}$ & 12 & 10 & 120 \\
\hline & & $15 \mathrm{t} /$ ha manure $+50 \mathrm{~N} 50 \mathrm{P}_{2} \mathrm{O}_{5} 50 \mathrm{~K}_{2} \mathrm{O}$ & 13 & 10 & 130 \\
\hline & & $20 \mathrm{t} /$ ha manure $+50 \mathrm{~N} 50 \mathrm{P}_{2} \mathrm{O}_{5} 50 \mathrm{~K}_{2} \mathrm{O}$ & 13 & 10 & 130 \\
\hline \multirow{8}{*}{3.2 . } & \multirow{8}{*}{$\begin{array}{c}\text { General expenses } \\
\text { (3.5\% of variable } \\
\text { expenses) }\end{array}$} & unfertilized & & & 26,28 \\
\hline & & $5 \mathrm{t} / \mathrm{ha}$ manure & & & 28,25 \\
\hline & & $10 \mathrm{t} / \mathrm{ha}$ manure & & & 30,20 \\
\hline & & $15 \mathrm{t} /$ ha manure & & & 32,17 \\
\hline & & 20 t/ha manure & & & 33,91 \\
\hline & & $10 \mathrm{t} /$ ha manure $+50 \mathrm{~N} 50 \mathrm{P}_{2} \mathrm{O}_{5} 50 \mathrm{~K}_{2} \mathrm{O}$ & & & 41,13 \\
\hline & & $15 \mathrm{t} /$ ha manure $+50 \mathrm{~N} 50 \mathrm{P}_{2} \mathrm{O}_{5} 50 \mathrm{~K}_{2} \mathrm{O}$ & & & 42,88 \\
\hline & & $20 \mathrm{t} /$ ha manure $+50 \mathrm{~N} 50 \mathrm{P}_{2} \mathrm{O}_{5} 50 \mathrm{~K}_{2} \mathrm{O}$ & & & 44,63 \\
\hline \multirow{5}{*}{ 3.6. } & \multirow{5}{*}{$\begin{array}{l}\text { TOTAL FIXE } \\
\text { EXPENSES }\end{array}$} & unfertilized & & & 126,28 \\
\hline & & $5 \mathrm{t} / \mathrm{ha}$ manure & & & 138,25 \\
\hline & & 10 t/ha manure & & & 140,20 \\
\hline & & 15 t/ha manure & & & 142,17 \\
\hline & & 20 t/ha manure & & & 153,91 \\
\hline
\end{tabular}




\begin{tabular}{|c|c|c|c|}
\hline & & $10 \mathrm{t} /$ ha manure $+50 \mathrm{~N} 50 \mathrm{P}_{2} \mathrm{O}_{5} 50 \mathrm{~K}_{2} \mathrm{O}$ & 161,13 \\
\hline & & $15 \mathrm{t} /$ ha manure $+50 \mathrm{~N} 50 \mathrm{P}_{2} \mathrm{O}_{5} 50 \mathrm{~K}_{2} \mathrm{O}$ & 172,88 \\
\hline & & $20 \mathrm{t} /$ ha manure $+50 \mathrm{~N} 50 \mathrm{P}_{2} \mathrm{O}_{5} 50 \mathrm{~K}_{2} \mathrm{O}$ & 174,63 \\
\hline \multirow{8}{*}{4.} & \multirow{8}{*}{$\begin{array}{c}\text { TOTAL } \\
\text { PRODUCTION } \\
\text { EXPENSES }\end{array}$} & unfertilized & 877,28 \\
\hline & & $5 \mathrm{t} / \mathrm{ha}$ manure & 945,25 \\
\hline & & $10 \mathrm{t} / \mathrm{ha}$ manure & 1003,20 \\
\hline & & $15 \mathrm{t} / \mathrm{ha}$ manure & 1061,17 \\
\hline & & 20 t/ha manure & 1122,91 \\
\hline & & $10 \mathrm{t} / \mathrm{ha}$ manure $+50 \mathrm{~N} 50 \mathrm{P}_{2} \mathrm{O}_{5} 50 \mathrm{~K}_{2} \mathrm{O}$ & 1336,13 \\
\hline & & $15 \mathrm{t} /$ ha manure $+50 \mathrm{~N} 50 \mathrm{P}_{2} \mathrm{O}_{5} 50 \mathrm{~K}_{2} \mathrm{O}$ & 1397,88 \\
\hline & & $20 \mathrm{t} /$ ha manure $+50 \mathrm{~N} 50 \mathrm{P}_{2} \mathrm{O}_{5} 50 \mathrm{~K}_{2} \mathrm{O}$ & 1449,63 \\
\hline
\end{tabular}

Source: own calculation

Table 2 summarizes the indicators and presents rates of profitability. Gross margin had a value of 3027.59 lei to variant 7, 2845.59 lei for variant 6, 2731.59 lei variant 5, 2709.59 lei variant 8. The variants 2, 3 and 4, did not exceed 2200 lei, while the witness was had a gross margin of 1517.59 lei.

Gross profit, calculated as the difference between gross margin and fixed costs, had good values for the last 4 variants (2854.71 lei - variant 7, 2684.46 lei - variant 6, 2577.68 lei - variant 5 and 2534.96 lei - variant 8), satisfactory for variants 3 and 4 (1917.39 and 2023.42 lei) and lower levels for witness and variant 2 (1391,31 - 1647,34 lei).

The gross profit rate exceeds $200 \%$ for variants 5, 6, 7 and have values lower than this limit for the other variants.

Net profit recorded for unfertilized variant was 1168.7 lei, increasing with the applied doses up to 2397.96 lei for fertilization by $15 \mathrm{t} /$ ha manure $+50 \mathrm{~N} 50 \mathrm{P}_{2} \mathrm{O}_{5} 50 \mathrm{~K}_{2} \mathrm{O}$, and starting to decline over this

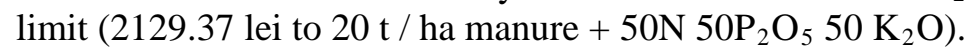

Table 2: Synthetic indicators

\begin{tabular}{|c|c|c|}
\hline Specification & Variant & Value ( Lei), \% \\
\hline \multirow{8}{*}{ Crude product } & unfertilized & 2268,59 \\
\hline & $5 \mathrm{t} /$ ha manure & 2592,59 \\
\hline & 10 t/ha manure & 2920,59 \\
\hline & $15 \mathrm{t} / \mathrm{ha}$ manure & 3084,59 \\
\hline & 20 t/ha manure & 3700,59 \\
\hline & $10 \mathrm{t} /$ ha manure $+50 \mathrm{~N}+50 \mathrm{P}_{2} \mathrm{O}_{5}+50 \mathrm{~K}_{2} \mathrm{O}$ & 4020,59 \\
\hline & $15 \mathrm{t} /$ ha manure $+50 \mathrm{~N}+50 \mathrm{P}_{2} \mathrm{O}_{5}+50 \mathrm{~K}_{2} \mathrm{O}$ & 4252,59 \\
\hline & $20 \mathrm{t} /$ ha manure $+50 \mathrm{~N}+50 \mathrm{P}_{2} \mathrm{O}_{5}+50 \mathrm{~K}_{2} \mathrm{O}$ & 3984,59 \\
\hline \multirow{8}{*}{ Variable expenses } & unfertilized & 751 \\
\hline & $5 \mathrm{t} /$ ha manure & 807 \\
\hline & $10 \mathrm{t} / \mathrm{ha}$ manure & 863 \\
\hline & $15 \mathrm{t} / \mathrm{ha}$ manure & 919 \\
\hline & 20 t/ha manure & 969 \\
\hline & $10 \mathrm{t} / \mathrm{ha}$ manure $+50 \mathrm{~N}+50 \mathrm{P}_{2} \mathrm{O}_{5}+50 \mathrm{~K}_{2} \mathrm{O}$ & 1175 \\
\hline & $15 \mathrm{t} /$ ha manure $+50 \mathrm{~N}+50 \mathrm{P}_{2} \mathrm{O}_{5}+50 \mathrm{~K}_{2} \mathrm{O}$ & 1225 \\
\hline & $20 \mathrm{t} /$ ha manure $+50 \mathrm{~N}+50 \mathrm{P}_{2} \mathrm{O}_{5}+50 \mathrm{~K}_{2} \mathrm{O}$ & 1275 \\
\hline \multirow{8}{*}{ Gross margin } & unfertilized & 1517,59 \\
\hline & $5 \mathrm{t} /$ ha manure & 1785,59 \\
\hline & 10 t/ha manure & 2057,59 \\
\hline & $15 \mathrm{t} /$ ha manure & 2165,59 \\
\hline & 20 t/ha manure & 2731,59 \\
\hline & $10 \mathrm{t} / \mathrm{ha}$ manure $+50 \mathrm{~N}+50 \mathrm{P}_{2} \mathrm{O}_{5}+50 \mathrm{~K}_{2} \mathrm{O}$ & 2845,59 \\
\hline & $15 \mathrm{t} /$ ha manure $+50 \mathrm{~N}+50 \mathrm{P}_{2} \mathrm{O}_{5}+50 \mathrm{~K}_{2} \mathrm{O}$ & 3027,59 \\
\hline & $20 \mathrm{t} /$ ha manure $+50 \mathrm{~N}+50 \mathrm{P}_{2} \mathrm{O}_{5}+50 \mathrm{~K}_{2} \mathrm{O}$ & 2709,59 \\
\hline \multirow{4}{*}{ Fixed expenses } & unfertilized & 126,28 \\
\hline & $5 \mathrm{t} /$ ha manure & 138,25 \\
\hline & $10 \mathrm{t} /$ ha manure & 140,20 \\
\hline & $15 \mathrm{t} / \mathrm{ha}$ manure & 142,17 \\
\hline
\end{tabular}




\begin{tabular}{|c|c|c|}
\hline & 20 t/ha manure & 153,91 \\
\hline & $10 \mathrm{t} /$ ha manure $+50 \mathrm{~N}+50 \mathrm{P}_{2} \mathrm{O}_{5}+50 \mathrm{~K}_{2} \mathrm{O}$ & 161,13 \\
\hline & $15 \mathrm{t} / \mathrm{ha}$ manure $+50 \mathrm{~N}+50 \mathrm{P}_{2} \mathrm{O}_{5}+50 \mathrm{~K}_{2} \mathrm{O}$ & 172,88 \\
\hline & $20 \mathrm{t} /$ ha manure $+50 \mathrm{~N}+50 \mathrm{P}_{2} \mathrm{O}_{5}+50 \mathrm{~K}_{2} \mathrm{O}$ & 174,63 \\
\hline \multirow{8}{*}{ Gross profit } & unfertilized & 1391,31 \\
\hline & $5 \mathrm{t} /$ ha manure & 1647,34 \\
\hline & 10 t/ha manure & 1917,39 \\
\hline & $15 \mathrm{t} / \mathrm{ha}$ manure & 2023,42 \\
\hline & 20 t/ha manure & 2577,68 \\
\hline & $10 \mathrm{t} /$ ha manure $+50 \mathrm{~N}+50 \mathrm{P}_{2} \mathrm{O}_{5}+50 \mathrm{~K}_{2} \mathrm{O}$ & 2684,46 \\
\hline & $15 \mathrm{t} /$ ha manure $+50 \mathrm{~N}+50 \mathrm{P}_{2} \mathrm{O}_{5}+50 \mathrm{~K}_{2} \mathrm{O}$ & 2854,71 \\
\hline & $20 \mathrm{t} /$ ha manure $+50 \mathrm{~N}+50 \mathrm{P}_{2} \mathrm{O}_{5}+50 \mathrm{~K}_{2} \mathrm{O}$ & 2534,96 \\
\hline \multirow{8}{*}{ Total expenditures } & unfertilized & 877,28 \\
\hline & $5 \mathrm{t} /$ ha manure & 945,25 \\
\hline & 10 t/ha manure & 1003,20 \\
\hline & $15 \mathrm{t} / \mathrm{ha}$ manure & 1061,17 \\
\hline & 20 t/ha manure & 1122,91 \\
\hline & $10 \mathrm{t} /$ ha manure $+50 \mathrm{~N}+50 \mathrm{P}_{2} \mathrm{O}_{5}+50 \mathrm{~K}_{2} \mathrm{O}$ & 1336,13 \\
\hline & $15 \mathrm{t} /$ ha manure $+50 \mathrm{~N}+50 \mathrm{P}_{2} \mathrm{O}_{5}+50 \mathrm{~K}_{2} \mathrm{O}$ & 1397,88 \\
\hline & $20 \mathrm{t} /$ ha manure $+50 \mathrm{~N}+50 \mathrm{P}_{2} \mathrm{O}_{5}+50 \mathrm{~K}_{2} \mathrm{O}$ & 1449,63 \\
\hline \multirow{8}{*}{$\begin{array}{c}\text { The gross profit rate } \\
\text { (\%) }\end{array}$} & unfertilized & 158,59 \\
\hline & $5 \mathrm{t} /$ ha manure & 174,27 \\
\hline & $10 \mathrm{t} / \mathrm{ha}$ manure & 191,12 \\
\hline & $15 \mathrm{t} / \mathrm{ha}$ manure & 190,67 \\
\hline & 20 t/ha manure & 229,55 \\
\hline & $10 \mathrm{t} /$ ha manure $+50 \mathrm{~N}+50 \mathrm{P}_{2} \mathrm{O}_{5}+50 \mathrm{~K}_{2} \mathrm{O}$ & 200,91 \\
\hline & $15 \mathrm{t} /$ ha manure $+50 \mathrm{~N}+50 \mathrm{P}_{2} \mathrm{O}_{5}+50 \mathrm{~K}_{2} \mathrm{O}$ & 204,21 \\
\hline & $20 \mathrm{t} / \mathrm{ha}$ manure $+50 \mathrm{~N}+50 \mathrm{P}_{2} \mathrm{O}_{5}+50 \mathrm{~K}_{2} \mathrm{O}$ & 174,86 \\
\hline \multirow{8}{*}{ Income tax } & unfertilized & 222,61 \\
\hline & $5 \mathrm{t} / \mathrm{ha}$ manure & 263,57 \\
\hline & $10 \mathrm{t} / \mathrm{ha}$ manure & 306,78 \\
\hline & $15 \mathrm{t} / \mathrm{ha}$ manure & 323,75 \\
\hline & 20 t/ha manure & 412,43 \\
\hline & $10 \mathrm{t} /$ ha manure $+50 \mathrm{~N}+50 \mathrm{P}_{2} \mathrm{O}_{5}+50 \mathrm{~K}_{2} \mathrm{O}$ & 429,51 \\
\hline & $15 \mathrm{t} /$ ha manure $+50 \mathrm{~N}+50 \mathrm{P}_{2} \mathrm{O}_{5}+50 \mathrm{~K}_{2} \mathrm{O}$ & 456,75 \\
\hline & $20 \mathrm{t} /$ ha manure $+50 \mathrm{~N}+50 \mathrm{P}_{2} \mathrm{O}_{5}+50 \mathrm{~K}_{2} \mathrm{O}$ & 405,59 \\
\hline \multirow{8}{*}{ Net profit } & unfertilized & 1168,7 \\
\hline & $5 \mathrm{t} / \mathrm{ha}$ manure & 1383,77 \\
\hline & $10 \mathrm{t} / \mathrm{ha}$ manure & 1610,61 \\
\hline & $15 \mathrm{t} / \mathrm{ha}$ manure & 1699,67 \\
\hline & 20 t/ha manure & 2165,25 \\
\hline & $10 \mathrm{t} / \mathrm{ha}$ manure $+50 \mathrm{~N}+50 \mathrm{P}_{2} \mathrm{O}_{5}+50 \mathrm{~K}_{2} \mathrm{O}$ & 2254,95 \\
\hline & $15 \mathrm{t} /$ ha manure $+50 \mathrm{~N}+50 \mathrm{P}_{2} \mathrm{O}_{5}+50 \mathrm{~K}_{2} \mathrm{O}$ & 2397,96 \\
\hline & $20 \mathrm{t} /$ ha manure $+50 \mathrm{~N}+50 \mathrm{P}_{2} \mathrm{O}_{5}+50 \mathrm{~K}_{2} \mathrm{O}$ & 2129,37 \\
\hline \multirow{8}{*}{$\begin{array}{c}\text { Net profit rate } \\
(\%)\end{array}$} & unfertilized & 133,22 \\
\hline & $5 \mathrm{t} /$ ha manure & 146,39 \\
\hline & $10 \mathrm{t} / \mathrm{ha}$ manure & 160,54 \\
\hline & $15 \mathrm{t} / \mathrm{ha}$ manure & 160,17 \\
\hline & 20 t/ha manure & 192,82 \\
\hline & $10 \mathrm{t} / \mathrm{ha}$ manure $+50 \mathrm{~N}+50 \mathrm{P}_{2} \mathrm{O}_{5}+50 \mathrm{~K}_{2} \mathrm{O}$ & 168,76 \\
\hline & $15 \mathrm{t} /$ ha manure $+50 \mathrm{~N}+50 \mathrm{P}_{2} \mathrm{O}_{5}+50 \mathrm{~K}_{2} \mathrm{O}$ & 171,54 \\
\hline & $20 \mathrm{t} /$ ha manure $+50 \mathrm{~N}+50 \mathrm{P}_{2} \mathrm{O}_{5}+50 \mathrm{~K}_{2} \mathrm{O}$ & 146,89 \\
\hline
\end{tabular}

Source: own calculation

Under this conditions, the net profit rate was maximum for variant fertilized with $20 \mathrm{t} / \mathrm{ha}$ manure (192,82\%), being followed by variant with $15 \mathrm{t} / \mathrm{ha}$ manure $+50 \mathrm{~N}+50 \mathrm{P}_{2} \mathrm{O}_{5}+50 \mathrm{~K}_{2} \mathrm{O}(171,54 \%)$, variant with 10 t/ha manure $+50 \mathrm{~N}+50 \mathrm{P}_{2} \mathrm{O}_{5}+50 \mathrm{~K}_{2} \mathrm{O}(168,76 \%)$ and variants fertilized only with manure in dose of 10 and $15 \mathrm{t} /$ ha $(160,54,160,17 \%)$. The rest of variants recorded weights of $146.89 \%$ for fertilizing with $20 \mathrm{t} / \mathrm{ha}+50 \mathrm{~N}+50 \mathrm{P}_{2} \mathrm{O}_{5}+50 \mathrm{~K}_{2} \mathrm{O}, 146.39 \%$ to $5 \mathrm{t} /$ ha manure and $133.22 \%$ to unfertilized. 


\section{Conclusions}

Taking into account the above mentioned regarding economic efficiency that the treatments have on permanent grassland of Agrostis capillaris in sub-mountainous area of Oltenia with chemical and organic fertilizers, or a combination of the two, we can formulate some conclusions as follows:

- Fertilizers and over-seeding increased the production obtained with 0,82 up to 4,96 tones (for variants 2 and 7), total gross product increasing from 2268,59 lei at unfertilized to 4252,59 lei for variant fertilized with $15 \mathrm{t} / \mathrm{ha}$ manure $+50 \mathrm{~N}+50 \mathrm{P}_{2} \mathrm{O}_{5}+50 \mathrm{~K}_{2} \mathrm{O}$;

- Total production expanses raged from 877,28 lei for witness up to 1449,63 lei for the variant fertilized with $20 \mathrm{t}$ /ha manure $+50 \mathrm{~N}+50 \mathrm{P}_{2} \mathrm{O}_{5}+50 \mathrm{~K}_{2} \mathrm{O}$;

- Gross margin knows a maximum for variant with $15 \mathrm{t} / \mathrm{ha}$ manure $+50 \mathrm{~N}+50 \mathrm{P}_{2} \mathrm{O}_{5}+50 \mathrm{~K}_{2} \mathrm{O}$ (3027,59 lei), important levels being recorded for variant with $10 \mathrm{t} / \mathrm{ha}$ manure $+50 \mathrm{~N}+50$ $\mathrm{P}_{2} \mathrm{O}_{5}+50 \mathrm{~K}_{2} \mathrm{O}$ (2845,59 lei), $20 \mathrm{t} / \mathrm{ha}$ manure (2731,59 lei) and $20 \mathrm{t} /$ ha manure $+50 \mathrm{~N}+50$ $\mathrm{P}_{2} \mathrm{O}_{5}+50 \mathrm{~K}_{2} \mathrm{O}$ (2709,59 lei) as well.;

- $\quad$ Net profit, after decreasing the incoming taxes, growth from 1168,7 for variant 1 to 2397,96 lei for variant $7\left(15 \mathrm{t} /\right.$ ha manure $\left.+50 \mathrm{~N}+50 \mathrm{P}_{2} \mathrm{O}_{5}+50 \mathrm{~K}_{2} \mathrm{O}\right)$, variants 5,6 and 8 having levels over 2000 lei also $\left(2129,37\right.$ lei for $20 \mathrm{t} /$ ha manure $+50 \mathrm{~N}+50 \mathrm{P}_{2} \mathrm{O}_{5}+50 \mathrm{~K}_{2} \mathrm{O}, 2165,25$ lei for $20 \mathrm{t} /$ ha manure and 2254,95 lei for $10 \mathrm{t} /$ ha manure $+50 \mathrm{~N}+50 \mathrm{P}_{2} \mathrm{O}_{5}+50 \mathrm{~K}_{2} \mathrm{O}$ ).

In 2014, the first year of experimentation, the variant with the highest net profit rate proved to be the one fertilized with $20 \mathrm{t}$ / ha manure.

\section{Acknowledgements}

This work was supported by the strategic grant POSDRU/159/1.5/S/133255, Project ID 133255 (2014), cofinanced by the European Social Fund within the Sectorial Operational Program Human Resources Development 2007-2013.

\section{References}

1. Ionescu, I., Permanent grassland in Northern Oltenia, Editors Universitaria, Craiova, Romania, (2001).

2. Medelete, D. M., PhD thesis, Research on technology and economic efficiency of grassland in the Subcarpathian side of Oltenia, Craiova (2009).

3. Pânzaru, R.L., Medelete, D.M., Gavril, Șt., Economy of crop production, Editors Universitaria, Craiova, Romania, (2009).

4. Pânzaru, R.L., Medelete, D.M., Gavril, Șt., 2007, Elements of management and marketing - Editors Universitaria, Craiova, Romania (2007). 